\title{
On Behavior Laplace Integral Operators with Generalized Bessel Matrix Polynomials and Related Functions
}

\author{
Muajebah Hidan $\mathbb{D}^{1}{ }^{1}$ Mohamed Akel, ${ }^{2}$ Salah Mahmoud Boulaaras, ${ }^{3,4}$ \\ and Mohamed Abdalla ${ }_{(\mathbb{D}}^{1,2}$ \\ ${ }^{1}$ Mathematics Department, Faculty of Science, King Khalid University, Abha 61471, Saudi Arabia \\ ${ }^{2}$ Mathematics Department, Faculty of Science, South Valley University, Qena 83523, Egypt \\ ${ }^{3}$ Department of Mathematics, College of Sciences and Arts, ArRas, Qassim University, Saudi Arabia \\ ${ }^{4}$ Laboratory of Fundamental and Applied Mathematics of Oran (LMFAO), University of Oran 1, Oran, 31000 Oran, Algeria
}

Correspondence should be addressed to Mohamed Abdalla; moabdalla@kku.edu.sa

Received 10 March 2021; Revised 12 April 2021; Accepted 20 April 2021; Published 30 April 2021

Academic Editor: Xinguang Zhang

Copyright (c) 2021 Muajebah Hidan et al. This is an open access article distributed under the Creative Commons Attribution License, which permits unrestricted use, distribution, and reproduction in any medium, provided the original work is properly cited.

Recently, the applications and importance of integral transforms (or operators) with special functions and polynomials have received more attention in various fields like fractional analysis, survival analysis, physics, statistics, and engendering. In this article, we aim to introduce a number of Laplace and inverse Laplace integral transforms of functions involving the generalized and reverse generalized Bessel matrix polynomials. In addition, the current outcomes are yielded to more outcomes in the modern theory of integral transforms.

\section{Introduction}

Recently, the integral transforms (or operators) have been extensively used tools in solving certain boundary value problems and certain integral equations. They are also useful in evaluating infinite integrals involving special functions or in solving differential equations of mathematical physics (see, e.g., [1-6] and the references cited therein). Laplace transform is a type of the integral transforms that is the most popular and widely used in several branches of astronomy, engineering, applied statistics, probability distributions, and applied mathematics (see, for instance, [7-13]).

A number of studies on the generalizations of Laplace transform associated with special polynomials have been contributed by Ortigueira and Machado [14], Jarad and Abdeljawad [15], Ganie and Jain [16], and Saifa et al. [17].

In 1949, Krall and Frink [18] introduced and discussed several properties of the generalized Bessel polynomials (GBPs), which are given by

$$
\mathfrak{V}_{n}(\alpha, \beta ; \xi)=\sum_{s=0}^{n}\left(\begin{array}{l}
n \\
s
\end{array}\right)(n+\alpha-1)_{s}\left(\frac{\xi}{\beta}\right)^{s}
$$

These polynomials, which seem to have been considered first by Bochner [19], are also mentioned in Romanovsky [20] and Krall [21].

Recently, these polynomials have been investigated in diverse ways and turned out to be applicable in a number of research fields (see, to exemplify, [22-25]).

Additionally, various extensions of the classical orthogonal polynomials to matrix setting were investigated. The matrix generalization of the generalized Bessel polynomials $\mathscr{B}_{n}^{\theta, \phi}(z), z \in \mathbb{C}$, for parameters (square) matrices $\theta$ and $\phi$, was also introduced in diverse ways ([26]; see also [27]). Various studies of the generalized Bessel matrix polynomials have been presented and discussed (see [27, 28]).

Recently, many works established Laplace integral transforms of special functions like Gauss's and Kummer's 
functions [29], generalized hypergeometric functions [30, 31], Aleph-Functions [32], and Bessel functions [33]. Whereas, some formulas corresponding to integral transforms of orthogonal matrix polynomials are little known and traceless in the literature. This motivates us to discuss Laplace integral transforms for functions involving generalized Bessel matrix polynomials. In particular, we obtain a number of useful Laplace and inverse Laplace type integrals of the generalized Bessel matrix polynomials together with ceratin elementary matrix functions, exponential function, logarithmic function, generalized hypergeometric matrix functions, and Bessel functions and products of generalized Bessel matrix polynomials. We also discuss some interesting and special cases of our main results.

\section{Preliminaries}

Here, we state some basic definitions and preliminaries which will be used in the article (see, for details, [34-36]).

Here and in the following sections, $C$ and $N$ denote the sets of complex numbers and positive integers, respectively, and $N_{0}=N \cup\{0\}$. We denote by $M_{r}(\mathbb{C})$ the space of $r \times r$ complex matrices endowed with classical norm defined by

$$
\|\theta\|=\sup _{y \neq 0}\left\{\frac{\|\theta y\|}{\|y\|}\right\}=\sup \{\|\theta y\|,\|y\|=1\} .
$$

This norm satisfies the inequality $\|\theta \phi\| \leq\|\theta\|\|\phi\|$, where $\theta$ and $\phi$ are in $M_{r}(\mathbb{C})$.

Definition 1. For any matrix $\theta$ in $M_{r}(\mathbb{C})$, the spectrum $\sigma(\theta)$ is the set of all eigenvalues of $\theta$ for which we denote

$\alpha(\theta)=\max \{\mathfrak{R}(\eta): \eta \in \sigma(\theta)\}$ and $\beta(\theta)=\min \{\mathfrak{R}(\eta): \eta \in \sigma(\theta)\}$,

where $\alpha(\theta)$ refers to the spectral abscissa of $\theta$ and for which $\beta(\theta)=-\alpha(-\theta)$. A matrix $\theta \in M_{r}(\mathbb{C})$ is said to be positive stable if and only if $\beta(\theta)>0$.

Definition 2 (see $[35,36]$ ). If $\theta \in M_{r}(\mathbb{C})$, and $w \in C$, then the matrix exponential $e^{\theta \omega}$ is given to be

$$
e^{\theta w}=I+\theta w+\cdots+\frac{\theta^{n}}{n !} w^{n}+\cdots
$$

where $I$ is the identity matrix in $M_{r}(\mathbb{C})$.

Definition 3 (see [37]). Let $\theta$ be a positive stable matrix in $M_{r}(\mathbb{C})$ with $\theta+n I$ is invertible for all integers $n \in N_{0}$, the Gamma matrix function $\Gamma(\theta)$ and the Digamma matrix function $\psi(\theta)$ are defined, respectively, as follows:

$$
\begin{aligned}
& \Gamma(\theta)=\int_{0}^{\infty} e^{-u} u^{\theta-I} d u ; \quad u^{\theta-I}=\exp ((\theta-I) \ln u) \\
& \psi(\theta)=\Gamma^{-1}(\theta) \Gamma^{\prime}(\theta)
\end{aligned}
$$

where $\Gamma^{-1}(\theta)$ and $\Gamma^{\prime}(\theta)$ are reciprocal and derivative of the Gamma matrix function.

Note that the scalar Gamma and Digamma functions are easily found when $r=1$ in (5) and (6), respectively (see, e.g., [38, Section 1.1])).

Definition 4 (see [?]). For all $\theta$ in $M_{r}(\mathbb{C})$, we assume

$$
\theta+k I \text { is invertible for all } k \in \mathbb{N}_{0} \text {, }
$$

and the Pochhammer symbol (the shifted factorial) is defined by

$$
(\theta)_{r}= \begin{cases}\theta(\theta+I) \cdots(\theta+(r-1) I)=\Gamma^{-1}(\theta) \Gamma(\theta+r I), & r \in \mathbb{N}, \\ I, & r=0 .\end{cases}
$$

Lemma 5 (see [34]). Let $\theta$ be a matrix in $M_{r}(\mathbb{C})$ such that $\|\theta\|<1$ and $\|I\|=1$. Then, $(I+\theta)^{-1}$ exists, and we have

$$
(I+\theta)^{-1}=I-\theta+\theta^{2}-\theta^{3}+\theta^{4}-\theta^{5}+\cdots
$$

Definition 6 (see [39]). Let $m$ and $n$ be finite positive integers, the generalized hypergeometric matrix function is given by

$$
{ }_{m} F_{n}(\theta ; \phi ; z)=\sum_{k=0}^{\infty} \prod_{i=1}^{m}\left(\theta_{i}\right)_{k} \prod_{j=1}^{n}\left[\left(\varphi_{j}\right)_{k}\right]^{-1} \frac{z^{k}}{k !}
$$

where $\theta_{i}, 1 \leq i \leq m$ and $\phi_{j}, 1 \leq j \leq n$ are commutative matrices in $M_{r}(\mathbb{C})$ with $\phi_{j}+k I$ are invertible for all integers $k \in N_{0}$ and $1 \leq i \leq m$. In [39], Abdalla discussed regions of convergence of (2.6).

Note that for $m=1, n=0$ in (10), we have the Binomial type matrix function ${ }_{1} F_{0}(\theta ;-; z)[39]$ as follows:

${ }_{1} F_{0}(\theta ;-; z)=(1-z)^{-\theta}=I+\theta z+\frac{\theta(\theta+I) z^{2}}{2 !}+\cdots+\frac{(\theta)_{n} z^{n}}{n !}+\cdots,|z|<1$.

Also, for $m=2, n=1$ in (10), we get the hypergeometric matrix function ${ }_{2} F_{1}$ (cf. [40]).

Further, the substitution $r=1$ in (10) leads to the classical generalized hypergeometric functions [38, Section 1.5], see also, [41].

Definition 7 (see [26]). Let $\theta$ and $\phi$ be commuting matrices in $M_{r}(\mathbb{C})$ such that $\phi$ is an invertible matrix. For any natural number $n \geq 0$, the $n^{\text {th }}$ generalized Bessel matrix polynomial $\mathscr{B}_{n}^{\theta, \theta}(z)$ is defined as 


$$
\begin{aligned}
\mathscr{B}_{n}^{\theta, \phi}(z) & =\sum_{r=0}^{n}\left(\begin{array}{c}
n \\
r
\end{array}\right)(\theta+(n-1) I)_{r}\left(z \phi^{-1}\right)^{r} \\
& =\sum_{r=0}^{n} \frac{(-1)^{r}}{r !}(-n I)_{r}(\theta+(n-1) I)_{r}\left(z \phi^{-1}\right)^{r} \\
& ={ }_{2} F_{0}\left(-n I, \theta+(n-1) I ;-;-z \phi^{-1}\right) .
\end{aligned}
$$

In addition, the $n^{\text {th }}$ reverse generalized Bessel matrix polynomial $\Theta_{n}^{(\theta, \phi)}(z)$ is given by (see [27])

$$
\begin{aligned}
\Theta_{n}^{(\theta, \phi)}(z)= & z^{n} \mathscr{B}_{n}^{\theta, \phi}\left(z^{-1}\right)=(-1)^{n} \Gamma^{-1}(-\theta-(2 n-2) I) \Gamma \\
& \cdot(-\theta+(n-2) I) \times{ }_{1} F_{1}(-n I ;-\theta-(2 n-2) I ; \phi z) \cdot \Theta_{n}^{(\theta, \phi)}(z) \\
= & z^{n} \mathscr{B}_{n}^{\theta, \phi}\left(z^{-1}\right)=(-1)^{n} \Gamma^{-1}(-\theta-(2 n-2) I) \Gamma \\
& \cdot(-\theta+(n-2) I) \times{ }_{1} F_{1}(-n I ;-\theta-(2 n-2) I ; \phi z) .
\end{aligned}
$$

Obviously, the $n^{\text {th }}$ generalized Bessel matrix polynomial $\mathscr{B}_{n}^{(\theta, \phi)}(z)$ when $r=1$ is easily found to be the scalar generalized Bessel polynomials (1.1).

Definition 8. Let $g(\tau)$ be a function of $\tau$ specified for $\tau>0$. Then, the Laplace transform of $g(\tau)$ is defined by

$$
\mathscr{G}(\lambda)=\mathscr{L}\{g(\tau): \lambda\}=\int_{0}^{\infty} e^{-\lambda \tau} g(\tau) d \tau, \quad \mathfrak{R}(\lambda)>0,
$$

provided that the improper integral exists, $e^{-\lambda u}$ is the kernel of the transformation and the function $g(\tau)$ is called the inverse Laplace transform of $\mathscr{G}(\lambda)$ (see [1, Chapter 3]; see also [7]).

The following Lemma, which may be easily derivable from (14), will be desired in the sequel.

Lemma 9. Let $\theta$ be a positive stable and invertible matrix in $M_{r}(\mathbb{C})$ and $\mathfrak{R}(\lambda)>0$. Then, we have

$$
\begin{aligned}
& \mathscr{L}\left\{\tau^{\theta}: \lambda\right\}=\int_{0}^{\infty} e^{-\lambda \tau} \tau^{\theta} d \tau=\lambda^{-(\theta+I)} \Gamma(\theta+I), \\
& \mathscr{L}\left\{\tau^{\theta}(\tau+1)^{-1}: \lambda\right\}=\Gamma(\theta+I) e^{\lambda} \Gamma(-\theta, \lambda),
\end{aligned}
$$

where $\Gamma(\theta, \lambda)$ is the incomplete Gamma matrix function [42].

$$
\begin{aligned}
\mathscr{L}\left\{g(\tau) e^{\theta \tau}: \lambda\right\} & =\mathscr{G}(\lambda I-\theta), \\
\mathscr{L}^{-1}\left\{\lambda^{-\theta}: \tau\right\} & =\tau^{(\theta-I)} \Gamma^{-1}(\theta) .
\end{aligned}
$$

\section{Laplace Type Integrals of Functions Involving $\mathscr{B}_{n}^{\theta, \phi}(z)$ and $\Theta_{n}^{\theta \cdot \phi}(z)$}

In this section, we investigate several Laplace-type transforms of functions involving generalized and reverse general- ized Bessel matrix polynomials asserted in the following theorems:

Theorem 10. Let $z, \lambda \in \mathbb{C}, \mathfrak{R}(\lambda)>0, n \in \mathbb{N}_{0}$, and $r \in \mathbb{N}$. Also, let $\theta, \phi$ and $A$ be matrices in $M_{r}(\mathbb{C})$ such that $\beta(A)>0$ and $\phi+k I$ are invertible for all $k \in \mathbb{N}_{0}$. For the function

$$
g_{1}(z)=z^{A-I} \mathscr{B}_{n}^{\theta, \phi}(z)
$$

we have

$\mathscr{G}_{1}(\lambda)=\mathscr{L}\left\{g_{1}(z): \lambda\right\}=\lambda^{-A} \Gamma(A)_{3} F_{0}\left[\begin{array}{cc}-n I, \theta+(n-1) I, A & \\ - & \end{array}\right]$

Proof. From the expansion series of the $\mathscr{B}_{n}^{\theta, \phi}(z)$ in (12) and upon using (15) in Lemma 9, we obtain

$$
\begin{aligned}
\mathscr{G}_{1}(\lambda) & =\sum_{s=0}^{n} \frac{(-n I)_{s}(\theta+(n-1) I)_{s}\left(-\phi^{-1}\right)^{s}}{s !} \mathscr{L}\left\{z^{A+(s-1) I}\right\} \\
& =\sum_{s=0}^{n} \frac{(-n I)_{s}(\theta+(n-1) I)_{s}\left(-\phi^{-1}\right)^{s}}{s !} \lambda^{-(A+s I)} \Gamma(A+s I) \\
& =\lambda^{-A} \Gamma(A) \sum_{s=0}^{n} \frac{(-n I)_{s}(\theta+(n-1) I)_{s}(A)_{s}\left(-(\lambda \phi)^{-1}\right)^{s}}{s !}
\end{aligned}
$$

Thus, we get the required result (19).

Theorem 11. Let $z, \lambda \in \mathbb{C}, \mathfrak{R}(\lambda)>0, n \in \mathbb{N}_{0}$, and $r \in \mathbb{N}$. Also, let $\theta, \phi$ and $A$ be matrices in $M_{r}(\mathbb{C})$ such that $\beta(A)>0, \phi+k I$ are invertible for all $k \in \mathbb{N}_{0}$ and $I-A$ satisfies the spectral condition (7). Further, let

$$
g_{2}(z)=z^{A-(n+1) I} \Theta_{n}(\theta, \phi ; z)
$$

Then,

$$
\left.\mathscr{G}_{2}(\lambda)=\mathscr{L}\left\{g_{2}(z): \lambda\right\}=\lambda^{-A} \Gamma(A)_{2} F_{1}\left[\begin{array}{c}
-n I, \theta+(n-1) I \\
I-A
\end{array}\right] \lambda \theta^{-1}\right]
$$

Proof. Starting from Definition 7, and applying the relation (15), it follows that 


$$
\begin{aligned}
\mathscr{G}_{2}(\lambda) & =\sum_{s=0}^{n} \frac{(-n I)_{s}(\theta+(n-1) I)_{s}\left(-\phi^{-1}\right)^{s}}{s !} \mathscr{L}\left\{z^{A-(s+1) I}\right\} \\
& =\sum_{s=0}^{n} \frac{(-n I)_{s}(\theta+(n-1) I)_{s}\left(-\phi^{-1}\right)^{s}}{s !} \lambda^{-(A-s I)} \Gamma(A-s I) \\
& =\lambda^{-A} \Gamma(A) \sum_{s=0}^{n} \frac{(-n I)_{s}(\theta+(n-1) I)_{s}\left[(I-A)_{s}\right]^{-1}\left(\lambda \phi^{-1}\right)^{s}}{s !} .
\end{aligned}
$$

Thus, the result (22) is established.

Theorem 12. Let $z, \mu, \lambda \in \mathbb{C}, \mathfrak{R}(\lambda-\mu)>0, n \in \mathbb{N}_{0}$, and $r \in \mathbb{N}$. Also, let $\theta, \phi$ and $A$ be matrices in $M_{r}(\mathbb{C})$ such that $\beta(A)>0$, $\phi+k I$ are invertible for all $k \in \mathbb{N}_{0}$ and $I-A$ satisfies the spectral condition (7). If

$$
g_{3}(z)=z^{A-I} e^{\mu z} \mathscr{B}_{n}^{\theta, \phi}\left(z^{-1}\right) .
$$

Then,

$\left.\mathscr{G}_{3}(\lambda)=\mathscr{L}\left\{g_{3}(z): \lambda\right\}=(\lambda-\mu)^{-A} \Gamma(A)_{2} F_{1}\left[\begin{array}{c}-n I, \theta+(n-1) I \\ I-A\end{array}\right](\lambda-\mu) \phi^{-1}\right]$.

Proof. For convenience, let the left-hand side of (25) be denoted by $S$ and by invoking the series expression of (12) to $S$, we obtain

$$
\begin{aligned}
\mathrm{S}= & \sum_{k=0}^{n} \frac{(-n I)_{s}(\theta+(n-1) I)_{k}\left(-\phi^{-1}\right)^{s}}{s !} \int_{0}^{\infty} z^{A-(s-1) I} e^{-(-\mu+\lambda) z} d z \\
= & \sum_{s=0}^{n} \frac{(-n I)_{s}(\theta+(n-1) I)_{s}\left(-\phi^{-1}\right)^{s}}{s !}(-\mu+\lambda)^{-(A-s I)} \Gamma(A-s I) \\
= & \Gamma(A)(\lambda-\mu)^{-A} \sum_{s=0}^{n}(-n I)_{s}(\theta+(n-1) I)_{s} \\
& \cdot\left[(I-A)_{s}\right]^{-1} \frac{\left((\lambda-\mu) \phi^{-1}\right)^{s}}{s !},
\end{aligned}
$$

therefore, (25) as desired.

Theorem 13. Let $z, w, \lambda \in \mathbb{C}, \mathfrak{R}(\lambda)>0, n \in \mathbb{N}_{0}$, and $r \in \mathbb{N}$. Also, let $\theta, \phi$ and $A$ be matrices in $M_{r}(\mathbb{C})$ such that $\beta(A)>0$ and $\phi+k I$ are invertible for all $k \in \mathbb{N}_{0}$. For the function

$$
g_{4}(z)=z^{A-I}(z+w)^{-1} \mathscr{B}_{n}^{\theta, \phi}(z)
$$

we have

$$
\begin{aligned}
\mathscr{G}_{4}(\lambda)= & \mathscr{L}\left\{g_{4}(z): \lambda\right\}=w^{A-I} \Gamma(A) e^{\lambda w} \\
& \times \sum_{k=0}^{n} \frac{(-n I)_{k}(\theta+(n-1) I)_{k}(A)_{k}}{k !} \Gamma \\
& \cdot(I-A-k I ; \lambda w)\left(-w \phi^{-1}\right)^{k},
\end{aligned}
$$

where $\Gamma(A, z)$ is the incomplete Gamma matrix function defined in [42].

Proof. To prove (28), we consider

$$
\begin{aligned}
\mathscr{G}_{4}(\lambda)= & \int_{0}^{\infty} z^{A-I}(z+w)^{-1} \mathscr{B}_{n}^{\theta, \phi}(z) e^{-\lambda z} d z \\
= & \sum_{k=0}^{n} \frac{(-n I)_{k}(\theta+(n-1) I)_{k}}{k !}\left(-\phi^{-1}\right)^{k} \\
& \times \int_{0}^{\infty} z^{A+(k-1) I}(z+w)^{-1} e^{-\lambda z} d z .
\end{aligned}
$$

According to (16) in Lemma 9, we get

$$
\begin{aligned}
\mathscr{G}_{4}(\lambda)= & \sum_{k=0}^{n} \frac{(-n I)_{k}(\theta+(n-1) I)_{k}}{k !} \Gamma(A+k I) \\
& \times w^{A+(k-1) I} e^{\lambda w} \Gamma((1-k) I-A, w \lambda)\left(-\phi^{-1}\right)^{k} \\
= & \Gamma(A) w^{A-I} e^{w \lambda} \sum_{k=0}^{n} \frac{(-n I)_{k}(\theta+(n-1) I)_{k}(A)_{k}}{k !} \\
& \times \Gamma((1-k) I-A, w \lambda)\left(-w \phi^{-1}\right)^{k} .
\end{aligned}
$$

This completes the proof of Theorem 13.

Theorem 14. Let $z, \lambda, v \in \mathbb{C}, \mathfrak{R}(\lambda)>0, \mathfrak{R}(v)>0, n, m \in \mathbb{N}_{0}$, and $r \in \mathbb{N}$. Also let $\theta, \phi$ and $A$ be matrices in $M_{r}(\mathbb{C})$ such that $\beta(A)>0, \phi+k I$ are invertible for all $k \in \mathbb{N}_{0},(1+n) I-A$ and $(2-n) I-A-\theta$ satisfies the spectral condition (7). Further, let

$$
g_{5}(z)=z^{A-I} \mathscr{B}_{n}^{\theta, \lambda I}\left(z^{-1}\right) \mathscr{B}_{m}^{v I, \phi}\left(z^{-1}\right)
$$

Then,

$$
\begin{aligned}
\mathscr{G}_{5}(\lambda)=\{ & \left.g_{5}(z): \lambda\right\}=\lambda^{-A} \Gamma(A) \Gamma(I-A) \Gamma(2 I-A-\theta) \times \Gamma^{-1} \\
& \cdot((1+n) I-A) \Gamma^{-1}((2-n) I-A-\theta) \times{ }_{3} F_{2} \\
& \cdot\left[\begin{array}{c}
-m I,(\nu+n-1) I, 2 I-A-\theta \\
; \lambda \phi^{-1} \\
(1+n) I-A,(2-n) I-A-\theta
\end{array}\right]
\end{aligned}
$$

Proof. To prove (32), we require the relation (15) and Definition 7 , thus we arrive at 


$$
\begin{aligned}
\mathscr{G}_{5}(\lambda)= & \sum_{s=0}^{n} \sum_{j=0}^{m} \frac{(-n I)_{s}(\theta+(n-1) I)_{s}\left(-\lambda^{-1}\right)^{s}}{s !} \\
& \times \frac{(-m I)_{j}(v I+(m-1) I)_{j}\left(-\phi^{-1}\right)^{j}}{j !} \mathscr{L}\left\{z^{A-(s+j+1) I}\right\} \\
= & \sum_{s=0}^{n} \sum_{j=0}^{m} \frac{(-n I)_{s}(\phi+(n-1) I)_{s}\left(-\lambda^{-1}\right)^{s}}{s !} \\
& \times \frac{(-m I)_{j}(v I+(m-1) I)_{j}\left(-\phi^{-1}\right)^{r}}{r !} \Gamma(A-(s+j) I) \lambda^{-(A-(s+j) I)} \\
= & \lambda^{-(A)} \Gamma(A) \sum_{j=0}^{m} \frac{(-m I)_{j}(v I+(m-1) I)_{j}\left(-\phi^{-1}\right)^{j}}{j !}\left[(I-A)_{j}\right]^{-1} \\
& \times \sum_{s=0}^{n} \frac{(-n I)_{s}(\phi+(n-1) I)_{s}\left[((1-j) I-A)_{s}\right]^{-1}}{s !} \\
= & \lambda^{-A} \Gamma(A) \Gamma(I-A) \Gamma(2 I-A-\theta)^{-1}(I-\theta+n I) \Gamma^{-1} \\
& \cdot(2 I-A-\theta-n I) \times \sum_{j=0}^{m} \frac{(-m I)_{j}(\nu I+(m-1) I)_{j}\left(-\lambda \phi^{-1}\right)^{j}}{j !} \\
& \cdot(2 I-A-\theta)_{j} \times\left[((1+n) I-A)_{r}\right]^{-1}\left[((2-n) I-A-\theta)_{j}\right]^{-1}
\end{aligned}
$$

This completes the proof of Theorem 14.

Theorem 15. Let $z, \lambda \in \mathbb{C}, \mathfrak{R}(\lambda)>0, n, m \in \mathbb{N}_{0}$, and $r \in \mathbb{N}$. Also, let $\theta, \vartheta, \phi$ and $A$ be matrices in $M_{r}(\mathbb{C})$ such that $\beta(A)$ $>0, \phi+k I$, are invertible for all $k \in \mathbb{N}_{0}, \vartheta,(\theta+A)$ and $\theta+A$

- I satisfies the spectral condition (7). Further, let

$$
g_{6}(z)=z^{A-I} \mathscr{B}_{n}^{\theta, \lambda z I}(1) \mathscr{B}_{m}^{\vartheta, \phi}(z) \mathscr{B}_{m}^{9, \phi}(-z)
$$

Then,

$$
\begin{aligned}
\mathscr{G}_{6}(\lambda)=\mathscr{L}\left\{g_{6}(z): \lambda\right\}=\frac{2^{A-I}}{\sqrt{\pi}}(\theta+A-I)_{n} \Gamma(A) \lambda^{-A}\left[(I-A)_{n}\right]^{-1} & \\
\times{ }_{8} F_{3} & {\left[\begin{array}{cc}
-m I, \vartheta+(m-1) I, \frac{1}{2}(\theta-I), \frac{1}{2} \vartheta, \frac{1}{2}(A+(1-n) I), & \\
\frac{1}{2}(A-n I), \frac{1}{2}(\theta+A+n I), \frac{1}{2}(\theta+A+(n-1) I) & \\
\vartheta I, \frac{1}{2}(\theta+A), \frac{1}{2}(\theta+A-I)
\end{array}\right] . }
\end{aligned}
$$

Proof. Applying the following formula (see [39])

$$
\mathscr{B}_{m}^{\vartheta, \phi}(z) \mathscr{B}_{m}^{\vartheta, \phi}(-z)={ }_{4} F_{1}\left[\begin{array}{c}
-m I, \vartheta+(m-1) I, \frac{1}{2}(\vartheta-I), \frac{1}{2} \vartheta \\
\vartheta-I
\end{array} ; 4 z^{2} \phi^{-2}\right] .
$$

We thus find that

$$
\begin{aligned}
\mathscr{G}_{6}(\lambda) & =\mathscr{L} \\
& \left.\left\{z^{A-I} \mathscr{B}_{n}^{\theta, \lambda z I}(1)_{4} F_{1}\left[\begin{array}{c}
-m I, \vartheta+(m-1) I, \frac{1}{2}(\vartheta-I), \frac{1}{2} \vartheta \\
\vartheta
\end{array}\right] z^{2} \phi^{-2}\right]\right\} \\
= & \sum_{s=0}^{n} \frac{(-n I)_{s}(\theta+(n-1) I)_{s}}{s !}\left(-\lambda^{-1}\right)^{s} \times \sum_{j=0}^{m} \frac{(-m I)_{j}(\vartheta+(m-1) I)_{j}}{j !} \\
& \cdot\left(\frac{1}{2}(\vartheta-I)\right)_{j}\left(\frac{1}{2} \vartheta\right)_{j}\left[(\vartheta-I)_{j}\right]^{-1}\left(4 \phi^{-2}\right)^{j} \times \mathscr{L}\left\{z^{A-(s+1+2 j) I}\right\} .
\end{aligned}
$$

Making use of (15), we observe that

$$
\begin{aligned}
& \mathscr{G}_{6}(\lambda)=\sum_{s=0}^{n} \frac{(-n I)_{s}(\theta+(n-1) I)_{s}}{s !}\left(-\lambda^{-1}\right)^{s} \\
& \times \sum_{j=0}^{m} \frac{(-m I)_{j}(\vartheta+(m-1) I)_{j}}{j !}\left(\frac{1}{2}(\vartheta-I)\right)_{j}\left(\frac{1}{2} \vartheta\right)_{j} \\
& \cdot\left[(\vartheta-I)_{j}\right]^{-1} \times\left(4 \phi^{-2}\right)^{j} \lambda^{-(A+(s-2 j) I)} \Gamma(A-(s-2 j) I) \\
& =\lambda^{-A} \Gamma(A) \sum_{j=0}^{m} \frac{(-m I)_{j}(\vartheta+(m-1) I)_{j}}{j !}\left(\frac{1}{2}(\vartheta-I)\right)_{j} \\
& \cdot\left(\frac{1}{2} \vartheta\right)_{j} \times\left[(\vartheta-I)_{j}\right]^{-1}(A)_{2 j}\left(4(\lambda \phi)^{-2}\right)^{j} \\
& =\sum_{s=0}^{n} \frac{(-n I)_{s}(\theta+(n-1) I)_{s}}{s !}\left[(I-A-2 j I)_{s}\right]^{-1} \\
& =\lambda^{-A} \frac{2^{A-I}}{\sqrt{\pi}} \Gamma(A) \Gamma(I-A) \Gamma(2 I-A-\theta) \Gamma^{-1} \\
& \cdot(I-A+n I) \Gamma^{-1}(2 I-A-\theta-n I) \\
& \times \sum_{j=0}^{m} \frac{(-m I)_{j}(\vartheta+(m-1) I)_{j}}{j !}\left(\frac{1}{2}(\vartheta-I)\right)_{r}\left(\frac{1}{2} \vartheta\right)_{j} \\
& \cdot\left(\frac{1}{2} A\right)_{j}\left[(\vartheta-I)_{j}\right]^{-1} \times\left(\frac{1}{2}(A+I)\right)_{j} \\
& \cdot\left(\frac{1}{2}(A+(1-n) I)\right)_{j}\left(\frac{1}{2}(A-n I)\right)_{j} \\
& \times\left(\frac{1}{2}(A+\theta+n I)\right)_{j}\left(\frac{1}{2}(A+\theta+(n-1) I)\right)_{j} \\
& \cdot\left[\left(\frac{1}{2}(A+I)\right)_{j}\right]^{-1} \times\left[\left(\frac{1}{2} A\right)_{j}\right]^{-1}\left[\left(\frac{1}{2}(A+\theta)\right)_{j}\right]^{-1} \\
& {\left[\left(\frac{1}{2}(A+\theta-I)\right)_{j}\right]^{-1} \cdot\left(16(\lambda \phi)^{-2}\right)^{j} \text {. }}
\end{aligned}
$$

Thus, after a simplification, we get the required result (35).

Theorem 16. Let $z, \lambda \in \mathbb{C}, \mathfrak{R}(\lambda)>0, n \in \mathbb{N}_{0}$, and $r \in \mathbb{N}$. Also, let $\theta, \phi$ and $A$ be matrices in $M_{r}(\mathbb{C})$ such that $\beta(A)>0$ and $\phi+k I$ are invertible for all $k \in \mathbb{N}_{0}$. For the function 


$$
g_{7}(z)=z^{A-I} \log z \mathscr{B}_{n}^{\theta, \phi}(z)
$$

then, we have

$$
\begin{aligned}
\mathscr{G}_{7}(\lambda)= & \mathscr{L}\left\{g_{7}(z): \lambda\right\}=\lambda^{-A} \Gamma(A) \sum_{s=0}^{n}(-n I)_{s}(\theta+(n-1) I)_{s}(A)_{s} \\
& \times \frac{\left(-(\lambda \phi)^{-1}\right)^{s}}{s !}(\psi(A+s I)-\log \lambda),
\end{aligned}
$$

where $\psi(A)$ is the Digamma matrix function defined in (6).

Proof. The proof of this Theorem is quite straight forward as

$$
\begin{aligned}
\mathscr{G}_{7}(\lambda)= & \int_{0}^{\infty} z^{A-I} \log z \mathscr{B}_{n}^{\theta, \phi}(z) e^{-\lambda z} d z \\
= & \sum_{s=0}^{n} \frac{(-n I)_{s}(\theta+(n-1) I)_{k}}{s !}\left(-\phi^{-1}\right)^{s} \\
& \times \int_{0}^{\infty} z^{A+(s-1) I} \log z e^{-\lambda z} d z .
\end{aligned}
$$

Upon using $(2,2)$, we have

$$
\Gamma(A+s I)=\int_{0}^{\infty} z^{A+(s-1) I} e^{-z} d z
$$

Hence,

$$
\Gamma^{\prime}(A+s I)=\int_{0}^{\infty} z^{A+(s-1) I} e^{-z} \log z d z
$$

We thus arrive at

$$
\begin{aligned}
\Psi(A+s I) & =\Gamma^{\prime}(A+s I) \Gamma^{-1}(A+s I) \\
& =\Gamma^{-1}(A+s I) \int_{0}^{\infty} z^{A+(s-1) I} e^{-z} \log z d z .
\end{aligned}
$$

Therefore, we get

$$
\begin{aligned}
\Psi(A+s I)= & \lambda^{A+s I} \Gamma^{-1}(A+s I) \int_{0}^{\infty} z^{A+(s-1) I} e^{-\lambda z} \log (\lambda z) d z \\
= & \lambda^{A+s I} \Gamma^{-1}(A+s I) \int_{0}^{\infty} z^{A+(s-1) I} e^{-\lambda z}[\log (\lambda)+\log (z)] d z \\
= & \lambda^{A+s I} \Gamma^{-1}(A+s I) \int_{0}^{\infty} z^{A+(s-1) I} e^{-\lambda z} \log (\lambda) d z \\
& +\lambda^{A+s I} \Gamma^{-1}(A+s I) \int_{0}^{\infty} z^{A+(s-1) I} e^{-\lambda z} \log (z) d z \\
= & \log (\lambda)+\lambda^{A+s I} \Gamma^{-1}(A+s I) \int_{0}^{\infty} z^{A+(s-1) I} e^{-\lambda z} \log (z) d z
\end{aligned}
$$

We thus have

$\int_{0}^{\infty} z^{A+(s-1) I} e^{-\lambda z} \log (z) d z=\lambda^{-(A+s I)} \Gamma(A+s I)[\Psi(A+s I)-\log \lambda]$.

From the above equations, we get the required result as follows:

$$
\begin{aligned}
\mathscr{G}_{7}(\lambda)= & \sum_{s=0}^{n} \frac{(-n I)_{s}(\theta+(n-1) I)_{s}(A)_{s}}{s !} \\
& \cdot\left(-(\lambda \phi)^{-1}\right)^{s} \times \lambda^{-A} \Gamma(A)[\Psi(A+s I)-\log \lambda] \\
= & \lambda^{-A} \Gamma(A) \sum_{s=0}^{n} \frac{(-n I)_{s}(\theta+(n-1) I)_{s}(A)_{s}}{s !} \\
& \times\left(-(\lambda \phi)^{-1}\right)^{s}[\Psi(A+s I)-\log \lambda] .
\end{aligned}
$$

Theorem 17. Let $z, \lambda \in \mathbb{C}, \mathfrak{R}(\lambda)>0, n, m, q \in \mathbb{N}_{0}$, and $r \in \mathbb{N}$. Also, let $\theta, \phi, E, D$ and $A$ be matrices in $M_{r}(\mathbb{C})$ such that $\beta(A)>0$, and $\phi+k I$ are invertible for all $k \in \mathbb{N}_{0}$. Further, let

$$
g_{8}(z)=z_{m}^{2 A-I} F_{q}\left(E ; D ; z^{2}\right) \mathscr{B}_{n}^{\theta, \phi}\left(z^{2}\right)
$$

Then,

$$
\begin{aligned}
\mathscr{G}_{8}(\lambda)= & \mathscr{L}\left\{g_{8}: \lambda\right\}=\frac{2^{2 A-I}}{\sqrt{\pi}} \Gamma(A) \Gamma\left(A+\frac{1}{2}\right) \lambda^{-2 A} \\
& \times \sum_{k=0}^{n} \frac{1}{k !}(-n I)_{k}(\theta+(n-1) I)_{k}(A)_{k}\left(A+\frac{1}{2}\right)_{k}\left(-4\left(\lambda^{2} \phi\right)^{-1}\right)^{k} \\
& \times{ }_{m+2} F_{q}\left(E, A+k I, A+\left(k+\frac{1}{2}\right) I ; D ; 4(\lambda)^{-2}\right),
\end{aligned}
$$

where ${ }_{m} F_{q}(E ; D ; z)$ is the generalized hypergeometric type matrix functions defined in (10) such that $\operatorname{Re}(\lambda)>0$ if $m<$ $q-1$ and $\operatorname{Re}(\lambda)>|\beta(A)|$ if $m=q-1$.

Proof. Using Definitions (10) and (12) and upon using (15), we obtain

$$
\begin{aligned}
\mathscr{G}_{8}(\lambda)= & \sum_{k=0}^{n} \frac{1}{k !}(-n I)_{k}(\theta+(n-1) I)_{k}\left(4(\phi)^{-1}\right)^{k} \\
& \times \sum_{r=0}^{\infty} \prod_{i=1}^{m}\left(E_{i}\right)_{r} \prod_{j=1}^{q}\left[\left(D_{j}\right)_{r}\right]^{-1} \frac{1}{k !} \mathscr{L}\left\{z^{2 A-(1-2 k-2 r) I}\right\} \\
= & \sum_{k=0}^{n} \frac{1}{k !}(-n I)_{k}(\theta+(n-1) I)_{k}\left(4(\phi)^{-1}\right)^{k} \\
& \times \sum_{r=0}^{\infty} \prod_{i=1}^{m}\left(E_{i}\right)_{r} \prod_{j=1}^{q}\left[\left(D_{j}\right)_{r}\right]^{-1} \frac{1}{k !} \times \lambda^{-2 A-(2 k+2 r) I} \Gamma \\
& \cdot(2 A+(2 k+2 r) I) .
\end{aligned}
$$


Thus, after a simplification, we obtain the result (49) in Theorem 3.11.

Theorem 18. Let $z, v, \sigma, \lambda \in \mathbb{C}, \mathfrak{R}(\lambda)>0, \mathfrak{R}(v)>-1, \mathfrak{R}(\sigma)$ $>0, n, m \in \mathbb{N}_{0}$, and $r \in \mathbb{N}$. Also, let $\theta$ be matrix in $M_{r}(\mathbb{C})$ such that $\beta(A)>0$ and $\phi+k I$ are invertible for all $k \in \mathbb{N}_{0}$. For the function

$$
g_{9}(z)=z^{v / 2} J_{v}\left(2(\sigma z)^{1 / 2}\right) \mathscr{B}_{n}^{\theta, \lambda z}(1)
$$

Then, we have

$$
\begin{aligned}
\mathscr{G}_{9}(\lambda)= & \mathscr{L}\left\{g_{9}(z): \lambda\right\}=\sigma^{v / 2}(\theta+v I)_{n}\left(\frac{1}{(-v)_{n}}\right) \lambda^{-(v+1)} \\
& \times{ }_{2} F_{2}\left[\begin{array}{cr}
1+v-m, \theta+(n+v) I \\
\\
1+v, \theta+v I
\end{array}\right],
\end{aligned}
$$

where $J_{v}(z)$ is the Bessel function of the first kind of order $v$ defined by (see, e.g., [38, 41, 43])

$$
J_{v}(z)=\sum_{s=0}^{\infty} \frac{(-1)^{s}}{s ! \Gamma(1+v+s)}\left(\frac{z}{2}\right)^{v+2 s} .
$$

Proof. According to (12) and (53) and upon sing (15), it follows that

$$
\begin{aligned}
\mathscr{G}_{9}(\lambda)= & \mathscr{L}\left\{z^{v / 2} J_{v}\left(2(\sigma z)^{1 / 2}\right) \mathscr{B}_{n}^{\theta, \lambda z}(1)\right\}=\sum_{m=0}^{\infty} \frac{(-1)^{m}(\sigma)^{m+(v / 2)}}{m ! \Gamma(1+v+m)} \\
& \times \sum_{k=0}^{n} \frac{(-n I)_{k}(\theta+(n-1) I)_{k}}{k !}\left(-\lambda^{-1}\right)^{k} \mathscr{L}\left\{z^{\frac{v}{2}+\frac{v}{2}-k+m}\right\} \\
= & (\sigma)^{v / 2} \sum_{m=0}^{\infty} \frac{(-\sigma)^{m}}{m ! \Gamma(1+v+m)} \times \sum_{k=0}^{n} \frac{(-n I)_{k}(\theta+(n-1) I)_{k}}{k !} \\
& \cdot\left(-\lambda^{-1}\right)^{k} \Gamma(1+v+m-k) \cdot \lambda^{v-m+k-1} \\
= & (\sigma)^{v / 2} \lambda^{v-1} \sum_{m=0}^{\infty} \frac{(-\sigma)^{m} \lambda^{-m} \Gamma(1+v+m)}{m ! \Gamma(1+v+m)} \\
& \times \sum_{k=0}^{n} \frac{(-n I)_{k}(\theta+(n-1) I)_{k}}{(-(v+m))_{k} k !}=(\sigma)^{v / 2} \lambda^{-v-1} \frac{(\theta+v I)_{n}}{(-v)_{n}} \\
& \cdot \sum_{m=0}^{\infty} \frac{(1+v-n)_{m}((v+n) I+\theta)_{m}\left[(\theta+v I)_{m}\right]^{-1}}{m !(1+v)_{m}}\left(\frac{-\sigma}{\lambda}\right)^{m} .
\end{aligned}
$$

This completes the proof of Theorem 18 .

\section{Inverse Laplace Type Integrals of Functions Involving $\mathscr{B}_{n}^{P, Q}(z)$}

Here, we obtain the following inverse Laplace type transforms of generalized Bessel matrix polynomials with products of some functions in the following theorem:
Theorem 19. Let $z, \lambda, \sigma \in \mathbb{C}, \mathfrak{R}(\lambda)>1 / 2|\mathfrak{R}(\sigma)|, n \in \mathbb{N}_{0}$, and $r \in \mathbb{N}$. Also, let $A$ be matrix in $M_{r}(\mathbb{C})$ such that $\beta(A)>$ o. If

$$
\mathscr{G}_{10}(\lambda)=\Gamma(A)\left(\lambda+\frac{1}{2} \sigma\right)^{-A} \mathscr{B}_{n}^{A-(n+1) I \frac{1}{\lambda+1 / 2 \sigma}}(-\sigma)
$$

Then,

$$
g_{10}(z)=z^{A-I} \exp \left(\frac{-1}{2} \sigma z\right)(1-\sigma z)^{n}
$$

Proof. It is sufficient to find Laplace transform of $g_{10}(z)$

$$
\begin{aligned}
\mathscr{G}_{10}(\lambda) & =\mathscr{L}\left\{z^{A-I} \exp \left(\frac{-1}{2} \sigma z\right)(1-\sigma z)^{n}\right\} \\
& \left.=\mathscr{L}\left\{z^{A-I} \exp \left(\frac{-1}{2} \sigma z\right)_{1} F_{0}(-n) \sigma z\right)\right\} \\
& =\sum_{k=0}^{n} \frac{(-n I)_{k} \sigma^{k}}{k !} \mathscr{L}\left\{z^{A-(1-k) I} \exp \left(\frac{-1}{2} \sigma z\right)\right\} \\
& =\sum_{k=0}^{n} \frac{(-n I)_{k} \sigma^{k}}{k !} \Gamma(A+k I)\left(\lambda+\frac{1}{2} \sigma\right)^{-(A+k I)} \\
& =\Gamma(A)\left(\lambda+\frac{1}{2} \sigma\right)^{-A} \sum_{k=0}^{n} \frac{(-n I)_{k}(A)_{k}}{k !}\left(\frac{\sigma}{(\lambda+1 / 2 \sigma)}\right)^{k},
\end{aligned}
$$

This finalizes the proof of Theorem 19.

Theorem 20. Let $z, \lambda, \sigma \in \mathbb{C}, \mathfrak{R}(\lambda)>0, \Re(\sigma)>0, n \in \mathbb{N}_{0}$, and $r \in \mathbb{N}$. Also, let $A$ be matrix in $M_{r}(\mathbb{C})$ such that $\beta(A+n I)>0$. Further, let

$$
\mathscr{G}_{11}(\lambda)=(-1)^{n} \sigma^{\frac{1}{2} A+n I} \lambda^{-(A+(2 n+1) I)} \exp \left(\frac{-\sigma}{\lambda} z\right) \mathscr{B}_{n}^{I-A-2 n I, \sigma}(\lambda) .
$$

Then,

$$
g_{11}(z)=z^{\frac{A}{2}+n I} J_{v}\left(2(\sigma z)^{1 / 2}\right) .
$$

Proof. By invoking to (15) and (53), we consider 


$$
\begin{aligned}
\mathscr{G}_{11}(\lambda)= & \mathscr{L}\left\{z^{\frac{A}{2}+n I} J_{v}\left(2(\sigma z)^{1 / 2}\right)\right\} \\
= & \sum_{r=0}^{\infty} \frac{\Gamma^{-1}(A+(1+r) I)(-\sigma)^{r} \sigma^{A / 2}}{r !} \mathscr{L}\left\{z^{A+(n+r) I}\right\} \\
= & \sigma^{A / 2} \Gamma^{-1}(A+I) \sum_{r=0}^{\infty} \frac{(-)^{r}\left[(A+I)_{r}\right]^{-1}}{r !} \Gamma \\
& \cdot(A+(r+n+1) I) \lambda^{-(A+(r+n+1) I)} \\
= & \sigma^{A / 2}(A+I)_{n} \lambda^{-(A+(n+1) I)} \exp \left(\frac{-\sigma}{\lambda}\right) \\
& \cdot \sum_{r=0}^{n} \frac{(-n I)_{r}\left[(A+I)_{r}\right]^{-1}}{r !}\left(\frac{\sigma}{\lambda}\right)^{r} \\
= & \sigma^{\frac{A}{2}+n I}(A+I)_{n} \lambda^{-(A+(2 n+1) I)} \exp \left(\frac{-\sigma}{\lambda}\right) \\
& \cdot \sum_{r=0}^{n} \frac{(-n I)_{r}\left[(A+I)_{r}\right]^{-1}}{r !}\left(\frac{\sigma}{\lambda}\right)^{r-n} \cdot
\end{aligned}
$$

Then,

$$
g_{13}(z)=\mathscr{B}_{n}^{\theta, \phi}\left(z^{-1}\right)
$$

Theorem 23. Let $z, \lambda, \mu \in \mathbb{C}, \mathfrak{R}(\lambda)>\mathfrak{R}(\mu)>0, n \in \mathbb{N}_{0}$, and $r \in \mathbb{N}$. Also let $\theta$ and $\phi$ be matrices in $M_{r}(\mathbb{C})$ such that $\phi+k$ $I$ are invertible for all $k \in \mathbb{N}_{0}$. Further, let

$$
\mathscr{G}_{14}(\lambda)=(\lambda-\mu)^{-1}{ }_{2} F_{0}\left[\begin{array}{c}
-n, \theta-(1-n) I \\
-
\end{array} ;(\lambda-\mu) \phi^{-1}\right] \text {. }
$$

Then,

$$
g_{14}(z)=\exp (\mu z) \mathscr{B}_{n}^{\theta, \phi}\left(z^{-1}\right)
$$

\section{Conclusion}

$$
\begin{aligned}
\mathscr{G}_{11}(\lambda)= & (-1)^{n} \sigma^{\frac{A}{2}+n I} \lambda^{-(A+(2 n+1) I)} \exp \left(\frac{-\sigma}{\lambda}\right) \\
& \times \sum_{k=0}^{n} \frac{(-n I)_{k}(-(A+n I))_{k}}{k !}\left(\frac{-\lambda}{\sigma}\right)^{k} \\
= & (-1)^{n} \sigma^{\frac{1}{2} A+n I} \lambda^{-(A+(2 n+1) I)} \exp \left(\frac{-\sigma}{\lambda} z\right) \mathscr{B}_{n}^{I-A-2 n I, \sigma}(\lambda) .
\end{aligned}
$$

This finalizes the proof of Theorem 20 .

The remaining results, which are given in the following theorems, can also be proven in a similar way. So we prefer to omit the details.

Theorem 21. Let $z, \lambda \in \mathbb{C}, \mathfrak{R}(\lambda)>0, n \in \mathbb{N}_{0}$, and $r \in \mathbb{N}$. Also, let $\theta$ and $\phi$ be matrices in $M_{r}(\mathbb{C})$ such that $\phi+k I$ are invertible for all $k \in \mathbb{N}_{0}$. Further, let

$$
\mathscr{G}_{12}(\lambda)=(-\phi)^{n} \lambda^{\theta+(2 n-2) I} \Gamma(2 I-\theta) \quad \mathscr{B}_{n}^{2 I-\theta-2 n I, \frac{(\phi-\lambda I)}{\lambda}}(-n) .
$$

Then,

$$
g_{12}(z)=z^{-(\theta+(n-1) I)} \mathscr{B}_{n}^{\theta, \phi}\left(z^{-1}\right)
$$

Theorem 22. Let $z, \lambda \in \mathbb{C}, \mathfrak{R}(\lambda)>0, n \in \mathbb{N}_{0}$, and $r \in \mathbb{N}$. Also let $\theta$ and $\phi$ be matrices in $M_{r}(\mathbb{C})$ such that $\phi+k I$ are invertible for all $k \in \mathbb{N}_{0}$. Further, let

$$
\mathscr{G}_{13}(\lambda)=\frac{1}{\lambda_{2}} F_{0}\left[\begin{array}{c}
-n, \theta-(n+1) I \\
-
\end{array} ; \lambda \phi^{-1}\right] \text {. }
$$

In fact, this work is a continuation of the recent paper by Abdalla [44]. In the current manuscript, the authors introduced various Laplace integral formulas of generalized Bessel matrix polynomials with certain elementary matrix functions, Binomial matrix functions exponential function, logarithmic function, generalized hypergeometric matrix functions, and Bessel function of the first kind. We also presented inverse Laplace transforms of generalized Bessel matrix polynomials with some functions. It is obvious that the results presented here which are involved in certain matrices in $M_{r}(\mathbb{C})$ may reduce to yield the corresponding scalar ones when $r=1$. Furthermore, the results derived in this article yields to many special cases; the interested reader may be referred to (see, e.g., $[1,7,45]$ ).

A remarkably large number of Laplace transforms and inverse Laplace transforms involving a variety of functions and polynomials have been presented (see, e.g., [45, pp. 129-299]). In this connection, we tried to give matrix versions of those outcomes for Laplace transforms and inverse Laplace formulas involving a variety of functions and polynomials (see, [45, pp. 129-299]).

\section{Data Availability}

No data were used to support the study.

\section{Conflicts of Interest}

This work does not have any conflicts of interest.

\section{Acknowledgments}

The fourth-named author extends their appreciation to the Deanship of Scientific Research at King Khalid University for funding work through research groups program under grant (R.G.P.1/3/42). 


\section{References}

[1] L. Debnath and D. Bhatta, Integral Transforms and Their Applications, Third Edition, Chapman and Hall (CRC Press), Taylor and Francis Group, London and New York, 2015.

[2] M. Consuelo Casabán, R. Company, V. Egorova, and L. Jódar, "Integral transform solution of random coupled parabolic partial differential models," Mathematical Methods in the Applied Sciences, vol. 43, no. 14, pp. 8223-8236, 2020.

[3] M. Kumar Bansal, D. Kumar, K. Nisar, and J. Singh, "Certain fractional calculus and integral transform results of incomplete $\aleph$-functions with applications," Mathematical Methods in the Applied Sciences, vol. 43, no. 8, pp. 5602-5614, 2020.

[4] A. Akdemir, S. Butt, M. Nadeem, and M. Ragusa, "New general variants of Chebyshev type Inequalities via generalized fractional integral operators," Mathematics, vol. 9, no. 2, p. 122, 2021.

[5] M. U. Din, M. Raza, and E. Deniz, "Univalence criteria for general integral operators involving normalized Dini functions," Univerzitet u Nišu, vol. 34, no. 7, pp. 2203-2216, 2020.

[6] A. Bakhet and F. He, "On 2-variables Konhauser matrix polynomials and their fractional integrals," Mathematics, vol. 8, no. 2, p. 232, 2020.

[7] J. Schiff, "The Laplace transform," in Theory and Applications, Springer, New York, 1999.

[8] D. Rani and V. Mishra, "Numerical inverse Laplace transform based on Bernoulli polynomials operational matrix for solving nonlinear differential equations," Results in Physics, vol. 16, article 102836, 2020.

[9] H. Srivastava, R. Agarwal, and S. Jain, "Integral transform and fractional derivative formulas involving the extended generalized hypergeometric functions and probability distributions," Mathematical Methods in the Applied Sciences, vol. 40, no. 1, pp. 255-273, 2017.

[10] D. Suthar, D. Kumar, and H. Habenom, "Solutions of fractional kinetic equation associated with the generalized multiindex Bessel function via Laplace transform," Differential Equations and Dynamical Systems, vol. 21, 2019.

[11] A. Apelblat, "Differentiation of the Mittag-Leffler functions with respect to parameters in the Laplace transform approach," Mathematics, vol. 8, no. 5, p. 657, 2020.

[12] D. Rani, V. Mishra, and C. Cattani, "Numerical inverse Laplace transform for solving a class of fractional differential equations," Symmetry, vol. 11, no. 4, p. 530, 2019.

[13] S. Viaggiu, "Axial and polar gravitational wave equations in a de Sitter expanding universe by Laplace transform," Classical and Quantum Gravity, vol. 34, no. 3, article 035018, 2017.

[14] M. Duarte Ortigueira and J. Tenreiro Machado, "Revisiting the 1D and 2D Laplace Transforms," Mathematics, vol. 8, no. 8, article 1330, 2020.

[15] F. Jarad and T. Abdeljawad, "Generalized fractional derivatives and Laplace transform," Discrete \& Continuous Dynamical Systems - S, vol. 13, no. 3, pp. 709-722, 2020.

[16] J. Ganie and R. Jain, "On a system of q-Laplace transform of two variables with applications," Journal of Computational and Applied Mathematics, vol. 366, article 112407, 2020.

[17] M. Saif, F. Khan, K. Sooppy Nisar, and S. Araci, "Modified Laplace transform and its properties," Journal of Mathematics and Computer Science, vol. 21, no. 2, pp. 127-135, 2020 .
[18] H. Krall and O. Frink, "A new class of orthogonal polynomials: the Bessel polynomials," Transactions of the American Mathematical Society, vol. 65, no. 1, pp. 100-115, 1949.

[19] S. Bochner, "Uber Sturm - Liouvillesche Polynomsysteme," Mathematische Zeitschrift, vol. 29, no. 1, pp. 730-736, 1929.

[20] V. Romanovsky, "Sur quelques classes nouvelles des polynômes orthogonaux," Comptes Rendus de l'Académie des Sciences - Series I - Mathematics, vol. 188, pp. 1023-1025, 1929.

[21] H. L. Krall, "Certain differential equations for Tchebycheff polynomials," Duke Mathematical Journal, vol. 4, no. 4, pp. 705-718, 1938.

[22] M. Altomare and F. Costabile, "A new determinant form of Bessel polynomials and applications," Mathematics and Computers in Simulation, vol. 141, pp. 16-23, 2017.

[23] M. Abdalla, M. Abul-Ez, and J. Morais, "On the construction of generalized monogenic Bessel polynomials," Mathematical Methods in the Applied Sciences, vol. 41, no. 18, pp. 93359348, 2018.

[24] D. Tcheutia, "Nonnegative linearization coefficients of the generalized Bessel polynomials," The Ramanujan Journal, vol. 48, no. 1, pp. 217-231, 2019.

[25] M. Izadi and C. Cattani, "Generalized Bessel polynomial for multi-order fractional differential equations," Symmetry, vol. 12, no. 8, p. 1260, 2020.

[26] Z. Kishka, A. Shehata, and M. Abul-Dahab, "The generalized Bessel matrix polynomials," The Journal of Mathematics and Computer Science, vol. 2, pp. 305-316, 2012.

[27] M. Abul-Dahab, M. Abul-Ez, Z. Kishka, and D. Constales, "Reverse generalized Bessel matrix differential equation, polynomial solutions, and their properties," Mathematical Methods in the Applied Sciences, vol. 38, no. 6, pp. 10051013, 2015.

[28] A. Shehata, "Certain generating matrix relations of generalized Bessel matrix polynomials from the view point of Lie algebra method," Bulletin of the Iranian Mathematical Society, vol. 44, no. 4, pp. 1025-1043, 2018.

[29] G. Milovanovic V., R. Parmar, and A. Rathie, "A study of generalized summation theorems for the series $2 \mathrm{~F} 1$ with an applications to Laplace transforms of convolution type integrals involving Kummer's functions 1F1," Applicable Analysis and Discrete Mathematics, vol. 12, no. 1, pp. 257-272, 2018.

[30] G. V. Milovanović, R. Parmar, and A. Rathie, "Certain Laplace transforms of convolution type integrals involving product of two special ${ }_{\mathrm{p}} \mathrm{F}_{\mathrm{p}}$ functions," Demonstratio Mathematica, vol. 51, no. 1, pp. 264-276, 2018.

[31] W. Koepf, I. Kim, and A. Rathie, "On a new class of Laplacetype integrals involving generalized hypergeometric functions," Axioms, vol. 8, no. 3, p. 87, 2019.

[32] A. Tassaddiq, A. Bhat, D. Jain, and F. Ali, "On $(p, q)$-Sumudu and $(p, q)$-Laplace transforms of the basic analogue of Alephfunction," Symmetry, vol. 12, no. 3, p. 390, 2020.

[33] R. T. Al-Khairy, "q-Laplace type transforms of q-analogues of Bessel functions," Journal of King Saud University - Science, vol. 32, no. 1, pp. 563-566, 2020.

[34] P. Lancaster, Theory of Matrices, Academic Press, New York, 1969.

[35] G. Golub and C. F. Van Loan, Matrix Computations, The Johns Hopkins University Press, MD, 1989.

[36] N. J. Higham, Functions of Matrices Theory and Computation, SIAM, USA, 2008. 
[37] J. C. Cortés, L. Jódar, F. J. Solís, and R. Ku-Carrillo, "Infinite matrix products and the representation of the matrix gamma function," Abstract and Applied Analysis, vol. 2015, Article ID 564287, 8 pages, 2015.

[38] H. M. Srivastava and J. Choi, Zeta and q-Zeta Functions and Associated Series and Integrals, Elsevier Science Publishers, Amsterdam, London and New York, 2012.

[39] M. Abdalla, "Further results on the generalised hypergeometric matrix functions," International Journal of Computing Science and Mathematics, vol. 10, no. 1, pp. 1-10, 2019.

[40] L. Jódar and J. C. Cortés, "On the hypergeometric matrix function," Journal of Computational and Applied Mathematics, vol. 99, no. 1-2, pp. 205-217, 1998.

[41] P. Agarwal, R. Agarwal, and M. Ruzhansky, Special Functions and Analysis of Differential Equations, 1st Edition, CRC Press, 2020.

[42] M. Abdalla, "On the incomplete hypergeometric matrix functions," The Ramanujan Journal, vol. 43, no. 3, pp. 663-678, 2017.

[43] S. Mondal and M. Akel, "Differential equation and inequalities of the generalized k-Bessel functions," Journal of Inequalities and Applications, vol. 2018, no. 1, 2018.

[44] M. Abdalla, "On Hankel transforms of generalized Bessel matrix polynomials," AIMS Mathematics, vol. 6, no. 6, pp. 6122-6139, 2021.

[45] A. Erdélyi, W. Magnus, F. Oberhettinger, and F. G. Tricomi, Tables of Integral Transforms Vol. I, McGraw-Hill Book Company, New York, Toronto and London, 1954. 